\title{
Bundesministerium für Familie, Senioren, Frauen und Jugend (BMFSFJ)
}

\author{
Dr. Jens Kreuter \\ Leiter des Arbeitsstabes Freiwilligendienste im BMFSFJ \\ jens.kreuter@bmfsfj.bund.de|www.bmfsfj.de
}

Ob im Krankenhaus oder bei Seniorinnen und Senioren, in der Kindertagesstätte oder im Umwelt- und Naturschutz, im Sport oder im kulturellen Bereich, in Deutschland oder irgendwo „draußen“ in der Welt: Menschen, die sich engagieren, bereichern unsere Gesellschaft, sie schenken Zeit, nehmen sich Zeit für Verantwortung und lassen sich für ihren weiteren Weg prägen.

In den vergangenen anderthalb Jahren ist es uns gelungen, die historische Chance zur Stärkung des freiwilligen Engagements zu nutzen. Mittlerweile sind wir mit insgesamt rund 85.000 Freiwilligen im Freiwilligen Sozialen Jahr (FSJ) und Freiwilligen Ökologischen Jahr (FÖJ), im neuen Bundesfreiwilligendienst (BFD) und den Auslandsfreiwilligendiensten einer neuen Kultur selbstverständlicher Freiwilligkeit näher als je zuvor. Über 38.000 Freiwillige sind im neuen Bundesfreiwilligendienst engagiert. Deutschlandweit stellen die ,älteren“ Freiwilligen („Ü 27“) im BFD einen erfreulichen Anteil von knapp 40 Prozent. Rund 20 Prozent der Freiwilligen sind sogar älter als 50 Jahre. Und auch die Jugendfreiwilligendienste gehen gestärkt aus diesen Entwicklungen hervor: Rund 50.000 junge Menschen leisten aktuell ein FSJ oder ein FÖJ. Dazu kommen die rund 6.000 Freiwilligen in den staatlich geförderten Auslandsfreiwilligendiensten - junge Menschen, die für freundschaftliche Beziehungen mit vielen Staaten der Welt einen wichtigen Beitrag leisten, die aber vor allem nach ihrer Rückkehr unsere Gesellschaft ungemein bereichern. Die Freiwilligendienste sind damit insgesamt aus der Nische herausgerückt und in der Mitte der Gesellschaft angekommen.

Ganz offensichtlich gibt es generationenübergreifend eine hohe Bereitschaft zum Engagement; darauf können wir sehr stolz sein. Mein großer Dank gilt den vielen Freiwilligen in allen Dienstformen, aber auch den Einrichtungen, Trägern und Verbänden, die Freiwilligen interessante Einsatzplätze anbieten, sie vorbereiten, begleiten und die Organisation des Dienstes übernehmen 
und nicht zuletzt all denjenigen, die ausländische Freiwillige, die bei uns einen Freiwilligendienst leisten, aufnehmen. Diese ideale Ausgangslage weiter auszubauen, die Chancen und Potentiale der Freiwilligendienste zu erkennen und mit allen gesellschaftlichen Akteuren zu nutzen, wird Aufgabe der nächsten Jahre sein.

Ich erhoffe mir von Voluntaris ein kreatives und unabhängiges Forum des Austausches über die vielen Herausforderungen in den verschiedenen Formaten. Ich wünsche mir eine Plattform zur fachlichen und sachlichen Auseinandersetzung mit Akteuren aus Wissenschaft, Praxis und Politik, aber auch den Blick auf die Grenzen der Freiwilligendienste und über sie hinaus. Ich erwarte einen spannenden Erfahrungsaustausch mit engagierten Vertreterinnen und Vertretern aus Wohlfahrtsverbänden, Kirchen und Zivilgesellschaft, mit Praktikerinnen und Praktikern aus Trägern und Einsatzstellen und, last but not least, mit Freiwilligen aller Altersgruppen und möglicher Dienstformen.

\title{
Bundesministerium für wirtschaftliche Zusammenarbeit und Entwicklung (BMZ)
}

\author{
Hans-Jürgen Beerfeltz
}

Staatssekretär des Bundesministeriums für wirtschaftliche Zusammenarbeit und Entwicklung |hans-juergen.beerfeltz@bmz.bund.de|www.bmz.de

Für Entwicklungszusammenarbeit ist das tatkräftige Engagement von Freiwilligen unverzichtbar. Denn: Der Staat kann die

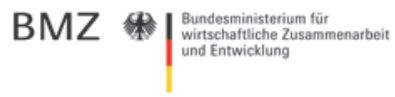
vielfältigen Herausforderungen nicht alleine bewältigen. Staat, Wirtschaft und die Zivilgesellschaft erfüllen je eigenständige Funktionen, die sich nicht wechselseitig substituieren lassen. Freiwillige bringen besondere Kreativität, Leidenschaft und Ausdauer mit. Zudem sind sie wichtige Multiplikatoren für die Anliegen der Entwicklungszusammenarbeit in der deutschen Gesellschaft: Sie erzählen ihren Familien, Freunden, Kolleginnen und Kollegen von ihren Erfahrungen. So wird Entwicklungszusammenarbeit auch erlebbar für jene, die noch nicht engagiert sind. 